\section{Diagnosis by endoscopic mucosal resection of a rare esophageal squamous cell carcinoma mimicking a subepithelial tumor}

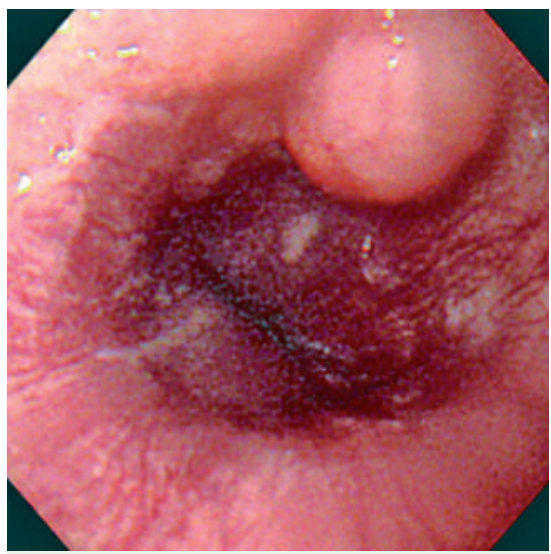

Fig. 1 Initial endoscopic view showing esophageal SET covered by normal-appearing mucosa.

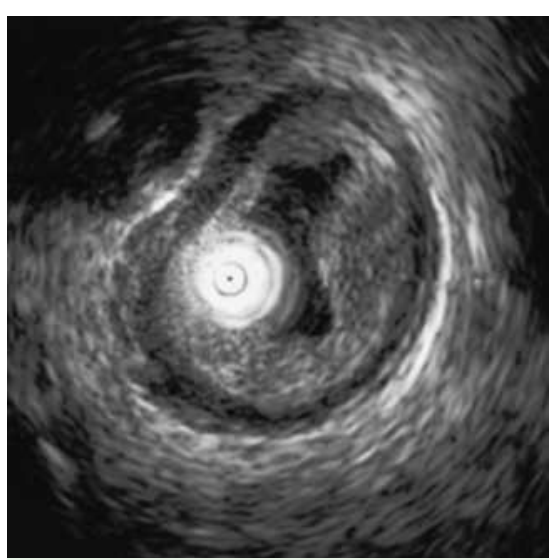

Fig. 2 Endosonographic image of the esophageal tumor showing a hypoechoic nodule in the submucosal layer.

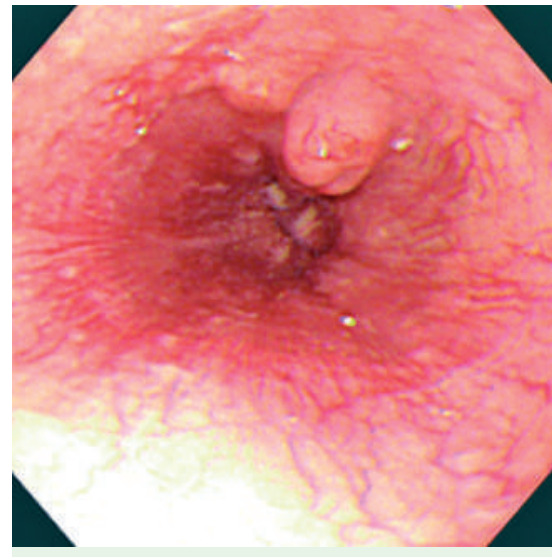

Fig. 3 Another small epithelial tumor seen adjacent to the first lesion after 14 months' follow-up.

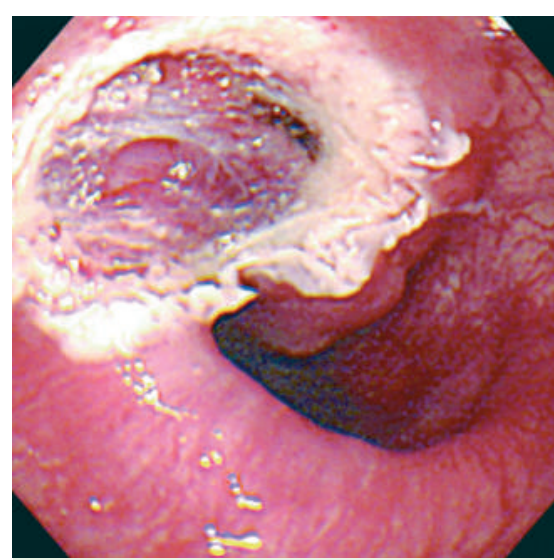

Fig. 4 Macroscopic view of residual tumor remaining at the resection margin after EMR-c.
A 48-year-old man was diagnosed with an esophageal subepithelial tumor (SET) found incidentally. Esophagogastroduodenoscopy (EGD) revealed a SET in the lower third of the esophagus $(36 \mathrm{~cm}$ away from the incisor) ( $\bullet$ Fig. 1). EUS confirmed a hypoechoic tumor, measuring $0.8 \mathrm{~cm}$, in the submucosal layer ( $\bullet$ Fig. 2). Benign leiomyoma was the initial impression, and hence no aggressive therapy was performed. The tumor size and appearance remained apparently unchanged during the following 2 years. The last EGD examination showed some alteration. Another smaller SET was also found adjacent to the previous lesion (๑ Fig. 3).

Endoscopic mucosal resection with the suction and cap method (EMR-c) was performed for tissue proof. A whitish tumor measuring $8 \mathrm{~mm}$ was discovered in the submucosal region of the specimen and residual tumor remained under macroscopic view ( Fig.4). The histological examination showed undifferentiated carcinoma. EMR-c was repeated 1 month later for a confirmatory diagnosis. Squamous cell carcinoma (SCC) was verified (๑ Fig.5). The patient therefore underwent esophagectomy and lymph node dissection. The final histological diagnosis was SCC invading the submucosal layer without lymph node metastasis or muscular layer invasion ( Fig.6). This was a rare case of esophageal SCC with an atypical presentation mimicking a SET. SETs of the esophagus are typically considered to be benign tumors [1]. In addition, histological diagnosis of esophageal SETs by biopsy is difficult. Therefore, SETs of the esophagus are frequently neglected unless symptoms occur [2].

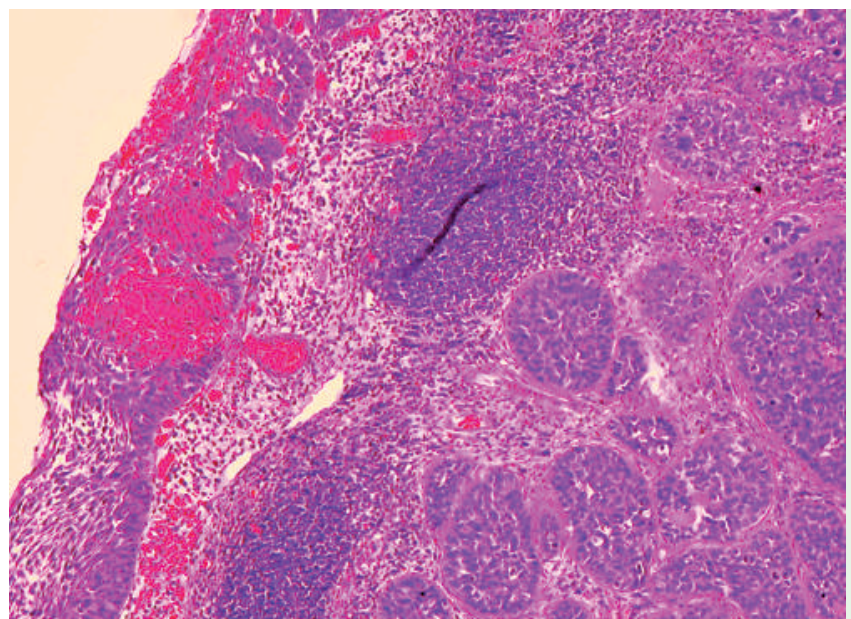

Fig. 5 Photomicrograph of EMR-c specimen showing malignant cells infiltrating the submucosal layer with glandular formation (H\&E, original magnification $\times 100$ ). 


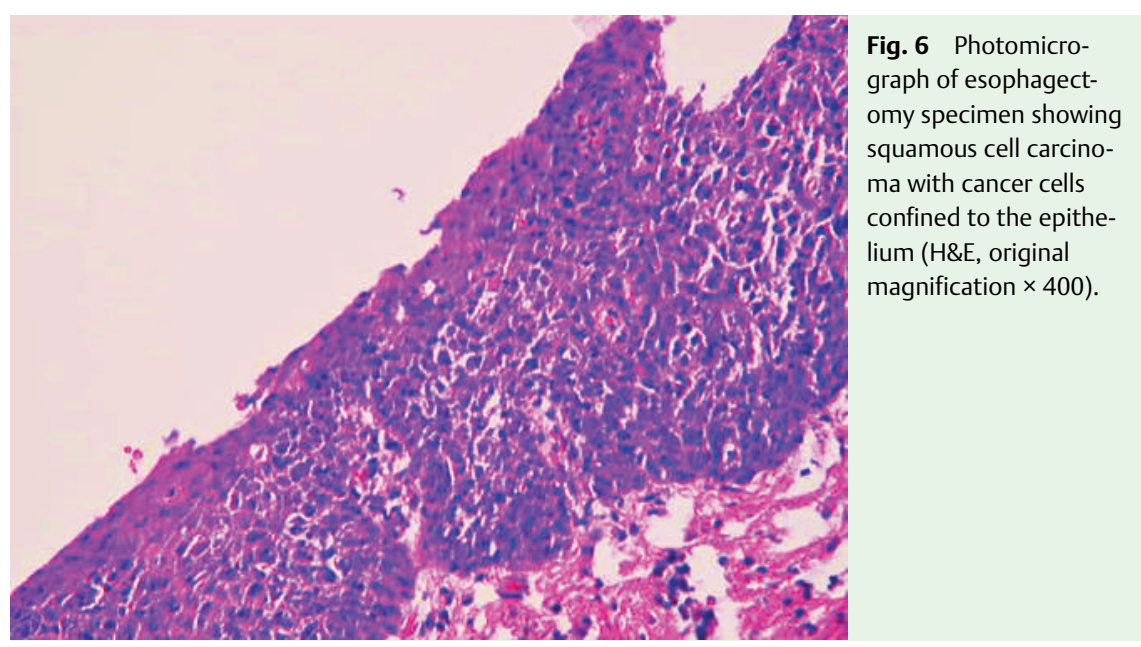

EMR-c, initially designed for resecting mucosal lesions, could be used for removing SETs of the gastrointestinal tract $[2-4]$. Tissue specimens gathered by the EMR-c method are generally large enough for immunohistochemical diagnosis. The method is convenient and safe for the resection of the SETs within the submucosal layer. On the basis of our experience, an esophageal SET can be an atypical presentation of SCC that should not be overlooked. Early endoscopic resection to get tissue proof is pivotal to avoid missing diagnosis.

Endoscopy_UCTN_Code_CCL_1AB_2AC_3AB

\section{References}

1 Hyun JH, Jeen YT, Chun HJ et al. Endoscopic resection of subepithelial tumor of the esophagus: results in 62 patients. Endoscopy $1997 ; 29: 165-170$

2 Lee I-L, Lin PY, Tung S-Y et al. Endoscopic submucosal dissection for the treatment of intraluminal gastric subepithelial tumors originating from the muscularis propria layer. Endoscopy 2006; 38: 1024-1028

3 Endo S, Hirasaki S, Doi T et al. Granular cell tumor occurring in the sigmoid colon treated by endoscopic mucosal resection using a transparent cap (EMR-C). [Review]. J. Gastroenterol 2003; 38: 385-389

4 Waxman I, Saltoh Y, Raju GS et al. High-frequency probe EUS-assisted endoscopic mucosal resection: a therapeutic strategy for subepithelial tumors of the GI tract. Gastrointest Endosc 2002; 55: 44-49

Bibliography

DOI 10.1055/s-2007-966666

Endoscopy 2007; 39: E301 -E302

( $)$ Georg Thieme Verlag KG Stuttgart · New York . ISSN 0013-726X

Corresponding author

I.-L. Lee, MD

Department of Gastroenterology

Chia-Yi Chang Gung Memorial Hospital

6, Section west

Chia-Po Road

Putz City

Chia-Yi 613

Taiwan

Fax: +886-5-3623005

giyilinlee@gmail.com 